\title{
Properties of tannin-glyoxal resins prepared from lyophilized and condensed tannin
}

\begin{abstract}
The main focus of this study was to prepare a thermoset adhesive using green way from two bio-product derivatives i.e., namely: condensed tannin (CT) and lyophilized tannin (LT) from grape pomace, which acquired from wine industries. Firstly, the obtained tannins were isolated and characterized especially by their thermal properties of the isolated tannin fractions which have been investigated in detail. Then, two adhesive resins were prepared using the isolated tannins with the glyoxal as crosslinking agents were employed to produce tannin-glyoxal resin. The evolution of the viscosity during the curing process of Resin-Tannin-Lyophilize-Glyoxal (RTLG) and Resin-Tannin-Condensed-Glyoxal (RTCG) was studied. The influence of the main gelation conditions such as temperature, $\mathrm{pH}$ and curing agent type on the gelation time was studied and discussed.
\end{abstract}

Keywords: grape pomace, tannins, gelation time, resin-tannin-lyophilize-glyoxal, resin-tannin-condensed-glyoxal
Volume 3 Issue 4 - 2017

\author{
Noureddine Baaka,' Mohamed Ammar, ${ }^{2}$ \\ Mohamed Khames Saad, ${ }^{2}$ Ramzi Khiari, ${ }^{1,3,4}$ \\ 'Faculty of Sciences, University of Monastir, Tunisia \\ ${ }^{2}$ Faculty of Sciences of Gafsa, Materials, Environment and Energy \\ Laboratory, University of Gafsa, Tunisia \\ 3Université Grenoble Alpes, France \\ ${ }^{4} \mathrm{CNRS}$, Laboratory of Papermaking Process Engineering, France
}

Correspondence: Ramzi Khiari, URI3 ES 63-Research Unity of Applied Chemistry and Environment, University of Monastir Monastir, Tunisia, Tel 2162006 0004, Fax 21673500278 , Email khiari_ramzi2000@yahoo.fr

Received: December 05, 2017 | Published: December 19, 2017
Abbreviations: CT, condensed tannins; LT, lyophilized tannins; TGA, thermo gravimetric analysis; RTLG, resin-tannin-lyofilise-glyoxal; RTCG, resin-tannin condensed-glyoxal

\section{Introduction}

Tannins are water-soluble polyphenolic compound, recalcitrant to biodegradation and with wide prevalence in plants. These compounds play important roles due to their resistant agents to microbial decomposition. In fact, it can be explain by the ability of these molecules to be inhibiting microbial growth by binding strongly to proteins and polysaccharides resembling cellulose and pectin.

From a chemical point of view, it is difficult to define tannins since the term encompasses some very diverse oligomers and polymers. ${ }^{1,2}$ It might be believed that the tannins are a heterogeneous group of high molecular weight of phenolic compound. Moreover, their capacity to form reversible and irreversible complexes with proteins, polysaccharides (cellulose, hemicellulose, pectin, etc.), alkaloids, nucleic acids and minerals. ${ }^{3,2}$

According to Pizzi research's, ${ }^{4}$ the term "tannin" has been frequently adopted to define two different groups of chemical compounds of phenolic nature namely: hydrolysable tannins and condensed tannins. Hydrolysable tannins can be considered polyesters derived from glucose. These can be classified into two categories specifically (i) gallotannins, which release gallic acid and its derivatives when submitted to acid hydrolysis; and (ii) ellagitannins, which upon hydrolysis release ellagic acid and valonic acid-ellagic acid being the most significant. ${ }^{5}$

(Figure 1) (Figure 2) illustrated an example of condensed tannins which consist of flavonoid units and more known as flavan-3ols (catechins) and flavan-3,4-diols (leucoantocyanidins)., ${ }^{4}$ The condensed tannins are most commonly presented as polymers, with an average degree of condensation ranging from 4 to 12 flavonoid units.

In recent years, tannins production has become a very important issue because of their increasing commercial interest in the field of pharmaceutical and food industries. In particular, tannins can be contribute to the therapeutic effects of some herbal medicines ; beverages rich in tannins have positive cardiovascular effects and, like some other smaller phenolic compounds, tannins may serve as dietary antioxidants. ${ }^{6,7}$

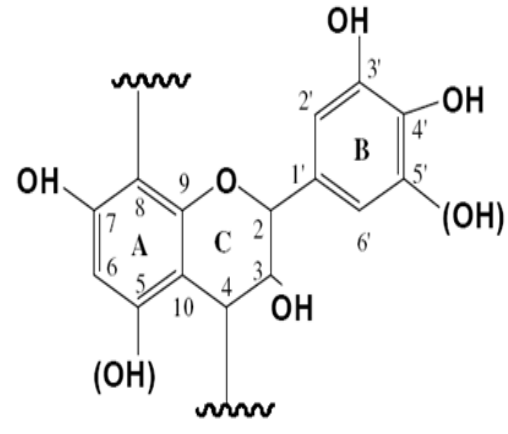

Figure I The chemical structure of condensed tannins.
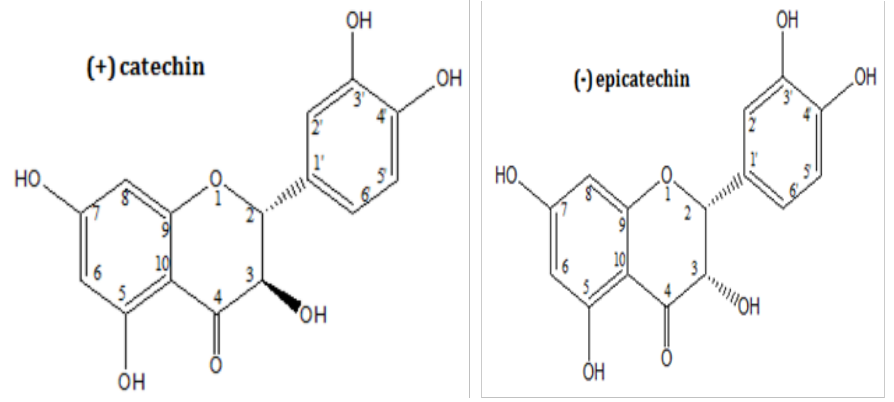

Figure 2 Structures of $(+)$ catechin and (-) epicatechin found in grape pomace.

Tannins have already been used commercially for 30years..$^{8-11}$ Condensed tannins are generally extracted from the bark of various trees. ${ }^{12,13}$ Elsewhere, grape is one of the world's largest fruit crops and this production generates a substantial volume of solid organic 
by-products. Only small amounts of these by products are up-graded or recycled; the pomace is used for animal feed or compost, without any pre-treatment. The remaining solid residues retain high levels of condensed tannins because of low extraction during winemaking. A few preliminary experiments concerning tannin extraction from grape pomace have been recently reported in previous communications. ${ }^{14,15}$

The aim of this study was to propose an economically and environmentally valuable utilization of grape pomace, wine industry by-products. Thus, extraction of tannins from this abundant resource was investigated using a process without any organic solvent or reagent. On the other hand, we successfully used tannins and glyoxal as inexpensive raw materials for preparing phenolic resin.

\section{Materials and methods}

\section{Grape residues}

Grape pomace was obtained by winemaker in Northern Tunisia, en 2014. They were a mixture of several grape varieties namely: Carignan and Alicante Bouchet of Vitis vinifera L. The mixture of stalks, seeds and skins was taken to the laboratory and dried at room temperature $\left(25 \pm 2^{\circ} \mathrm{C}\right)$. Subsequently the dried material was ground and a fine powder was prepared.

\section{Tannins extraction}

Grape pomace was treated with an aqueous solution of hydroxide sodium $(\mathrm{NaOH}) 5 \%(\mathrm{w} / \mathrm{w})$, with a solid-to-liquid ratio of $1: 10$. The extraction of tannins was carried out by heating at $10^{\circ} \mathrm{C}$ during $120 \mathrm{~min}$ in closed flasks using an Ahiba machine (laboratory machine: Ahiba Datacolor International, USA). After heating step, the pomace was then cooled, washed and filtered through filter paper. In order to isolate the tannin (Figure 3): two different processes were established after the extraction step namely:

a. Path 1: addition of dilute $\mathrm{HCl}$ to adjust the $\mathrm{pH}$ of the liquid phase reach 1.5 to precipitate the tannins which are collected by centrifugation at $8,000 \mathrm{rpm}$ for $15 \mathrm{~min}$ and then air-dried;

b. Path 2: During this process, a lyophilization method of the liquid phase to yield a crude residue was investigated.

All the chemicals were purchased from Sigma-Aldrich and were used without further purification.

\section{Resin synthesis}

During this work, two resins were condensed in a $6 \mathrm{dm}^{3}$ glass reactor equipped with a stirrer, a condenser, and external cooling with internal heating units. The Modified resins as represented in Table 1 were synthesized according to the preparation method reported by Mansouri et al. ${ }^{16}$ and Ammar et al. ${ }^{17}$ The curing fraction was 50\% of the amounts of Tannin, which is two grams for all samples. In the meantime, $1.5 \mathrm{~g}$ of sodium hydroxide (on dry basis) was added very progressively in the form of $30 \mathrm{wt} \%$ aqueous solution and mixed for $45 \mathrm{~min}$. After that, the mixture was heated at $97^{\circ} \mathrm{C}$ under mechanical stirring, for $2 \mathrm{~h}$. Then, the solution was cooled to room temperature. As recommended by the various standards used, all the experiments were duplicated and the differences between the two values were within an experimental error of $5 \%$.

\section{Gel point measurements}

The gel formation was studied by means of rheological measurements performed in an MCR 301 rheometer (Germany) with parallel plates of $25 \mathrm{~mm}$ diameter and samples of approximately $5 \mathrm{~mm}$ diameter with $1 \mathrm{~mm}$ thickness. The gelation point was determined by the divergence of viscosity, measured under a shear rate of $1 \mathrm{~s}^{-1}$. Then, the sample was cooled $\left(\mathrm{T}=4^{\circ} \mathrm{C}\right)$ and submitted again to a dynamic scan at a heating rate of $10^{\circ} \mathrm{Cmin}^{-1}$, using DSC. ${ }^{18-20}$ The test was repeated at least three times.

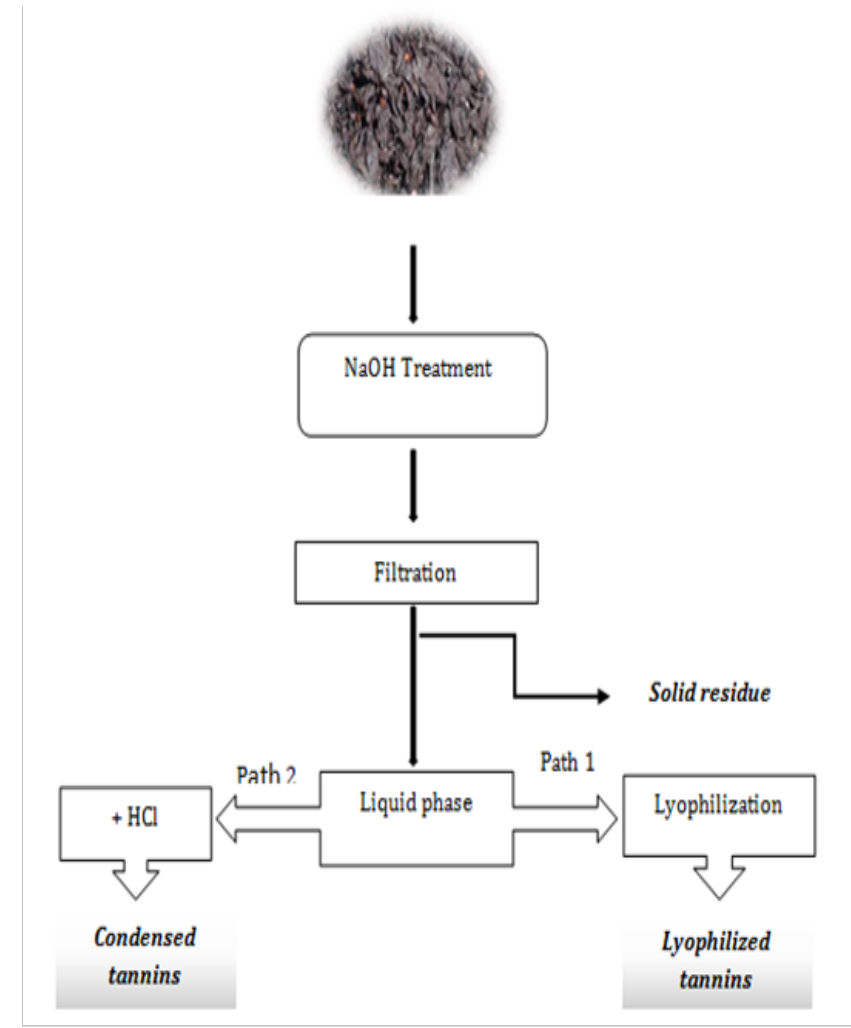

Figure 3 Schematic representation of the different Tannins prepared from grape pomace.

\section{Thermo gravimetric analysis (TGA)}

The thermal properties of obtained resins were performed using the thermo gravimetric analysis (TGA, Perkin-Elmer Pyris 1 TGA7 , USA). The test was done by introducing $10 \mathrm{mg}$ of cured RTLG or RTCG into a platinum sample pan and heated from 30 to $900^{\circ} \mathrm{C}$ at a heating rate of $10^{\circ} \mathrm{C} \cdot \mathrm{min}^{-1}$ under a nitrogen atmosphere with a constant flow rate of $20 \mathrm{~mL} \cdot \mathrm{min}^{-1}$. The analysis was repeated in duplicate.

\section{UV-visible spectra}

The UV spectra of the various tannins were recorded on an ultraviolet/visible spectrophotometer (Shimadzu double-beam spectrophotometer UV 1800). The different fraction lignin sample $(5 \mathrm{mg})$ was dissolved in $95 \%(\mathrm{v} / \mathrm{v})$ dioxane aqueous solution $(10 \mathrm{~mL})$. About $1 \mathrm{~mL}$ aliquot was diluted to $10 \mathrm{~mL}$ with $50 \%(\mathrm{v} / \mathrm{v})$ dioxane aqueous solution and the absorbance between 250 and $400 \mathrm{~nm}$ was recorded and measured. The measurement test was done at least five times.

\section{FTIR spectra}

The FTIR spectra were performed using a Bio-Rad spectrophotometer with a resolution of $4 \mathrm{~cm}^{-1}$ and scanning a wave length range from 500 to $4000 \mathrm{~cm}^{-1}$. $\mathrm{KBr}$-based solid pellets made of 
a suspension of $1 \mathrm{mg}$ of the material under investigation and $100 \mathrm{mg}$ of anhydrous $\mathrm{KBr}$.

\section{BET measurement}

BET (Brunauer-Emmett-Teller) surface analysis was performed on a TRISTAR II 3020 surface analyzer using the multipoint method. Tannin $(5 \mathrm{~g})$ were degassed at $120^{\circ} \mathrm{C}$ for $12 \mathrm{hr}$ and nitrogen adsorptiondesorption isotherms determined at $77 \mathrm{~K}$. Total pore volume was evaluated from the amount of nitrogen adsorbed at $\mathrm{P} / \mathrm{P} 0=0.99(\mathrm{P}$ is applied pressure, $\mathrm{P} 0$ is system initial pressure).

\section{Results and discussion}

\section{Isolation of Tannin}

As illustrated in the Figure 3, the tannins were extracted from grape pomace in basic medium using two ways according to Figure 3. Two different processes were used for tannins recovery:

a. Path 1: addition of dilute $\mathrm{HCl}$ to precipitate the tannins, which are collected by centrifugation and then air-dried.

b. Path 2: lyophilization of the liquid phase to yield a crude residue.

UV- Visible: UV-visible light absorption measurements have been used to semi-quantitatively assess the purity of tannin samples. The intensity of the absorbance is related to the level of tannin concentration and is proportional to the purity level of tannin. Therefore, a lower absorbance indicates the presence of non-tannin material such as carbohydrates. Figure 4 presents the UV spectra of CT and LT, the maximum absorbance for the two tannin samples occurred at $282 \mathrm{~nm}$, which originated from the phenolic groups in the tannin. Interestingly, as shown in the spectra, the highest absorbance values occurred in the CT samples, suggesting that these were the most pure tannin preparations. LT samples likely contained higher amounts of bound carbohydrate and were less pure.

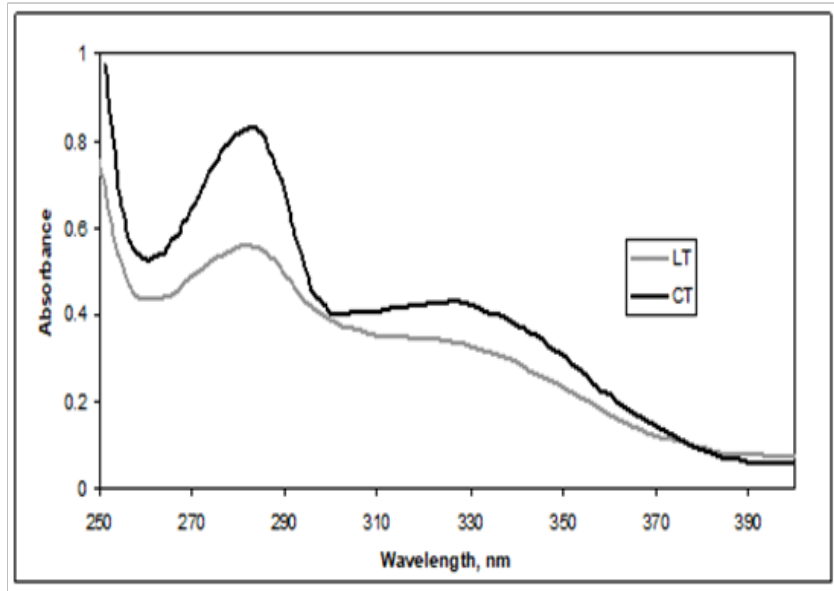

Figure 4 UV spectra of CT and LT.

FTIR Spectra: The FT-IR spectra of lyophilized tannins (LT) and condensed tannins (CT) were given in Figure 5 . The band at $3318 \mathrm{~cm}^{-1}$ was assigned to hydroxyl both contribute to phenolic and sugar content. The bands at $2973 \mathrm{~cm}^{-1}$ were due to $\mathrm{C}-\mathrm{H}$ stretching vibration assigned to methyl and methylene groups. A weak signal at $1878 \mathrm{~cm}^{-1}$ is assigned to carbonyl groups. The presence of carbonyl groups could be rationalized by the catechinic acid rearrangement occuring during the extraction process. The formation of catechinic acid is to be mini- mized because it should decrease the reactivity of the extract toward cross-linking agent. The bands at $1308 \mathrm{~cm}^{-1}, 1264 \mathrm{~cm}^{-1}$ and $1212 \mathrm{~cm}^{-1}$ were assigned to saturated C-C stretching attribute to CR2-CHR-CR structure caused by the opening of the pyran ring during acidification of flavonoid tannins. ${ }^{17}$ This reaction has a positive effect because it increases the solubility of tannins and reduces the viscosity of the extracts allowing the use of adhesive solutions of higher resin content. The condensed tannins displayed a different pattern: (i) No obviously band among $1308-1212 \mathrm{~cm}^{-1}$ was detected assigned to saturated $\mathrm{C}-\mathrm{C}$ stretching attribute to CR2-CHR-CR structure caused by the opening of the pyran ring in the spectra. (ii) An intense signal at about $1878 \mathrm{~cm}$ ${ }^{1}$ assigned to carbonyl groups indicates higher catechnic acid content in condensed tannins, the bands assigned to aromatic ring vibration $\left(1600-1400 \mathrm{~cm}^{-1}\right)$ are quite stronger than lyophilized tannin extract. These results are in accordance with the catechinic acid rearrangement occurred during the acidification step.

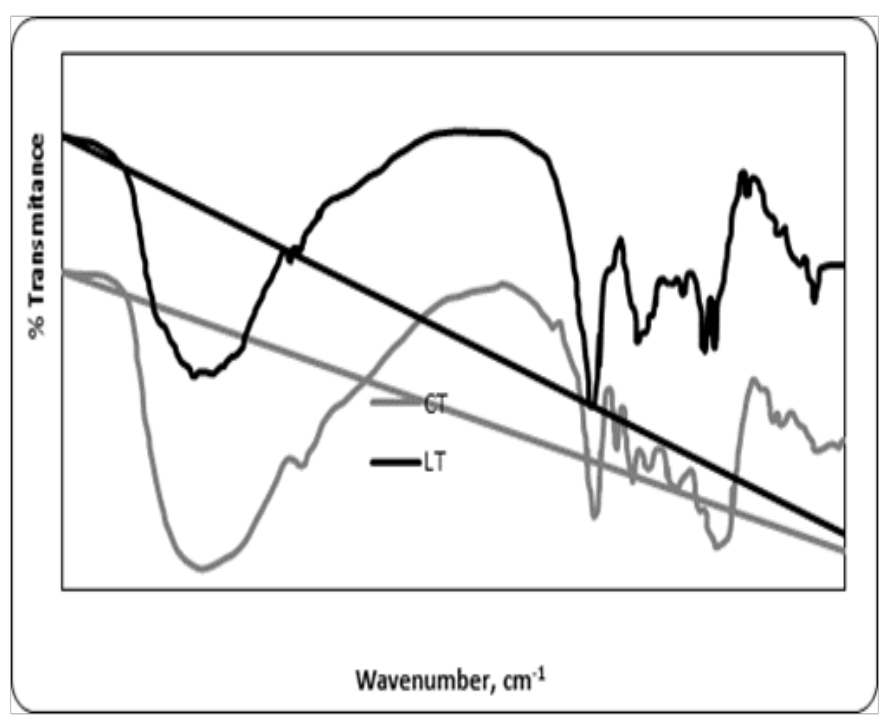

Figure 5 FTIR spectra of condensed tannins (CT) and Lyophilized tannins (LT).

Thermo gravimetric analysis (TGA): Thermo gravimetric analysis (TGA) was applied to determine the thermal stability and degradation of tannin. The TGA curve presents the weight loss percentage of the tannin extracts and the DTG cure is the differential curve, presents the mass lost rate. The thermal properties of the CT and LT fractions were studied used TGA. The results are showed in Figure 6. The weight lost is mainly composed by two steps. The mass lost of first step is attributed to the mass lost of water and some easy-degraded small low molecular materials such as simple sugars, organic acid, phenol and pigment. The secondly steps which is the mainly degradation step of tannin samples from 200 to $400^{\circ} \mathrm{C}$. The mass lost is nearly $33 \%$. It is attributed to the mass lost of polyphenol materials in the tannin extracts. It appears that an obvious difference occured in the first step (room temperature to $200^{\circ} \mathrm{C}$ ): concerning $\mathrm{CT}$, the mass lost nearly $9 \%$, mainly occurred by the evaporated of water content. It never appears apparent mass lost rate point compared to lyophilized tannins. This phenomenon can be attributed to the lower non-tannin fraction (such as low molecule sugars) in CT. This result is in agreement with UV spectra. We also observed that the CT mass loss temperature is higher than LT, this observation can be rationalized by a higher degree of polymerization of CT compared to LT.

Specific surface areas: Table 2 also summarizes specific surface 
areas, pore volumes and pore diameters of the two tannin obtained by the BET method. The average pore diameters as determined by BJH method were 10.4 and $3.8 \mathrm{~nm}$ for lyophilized and condensed tannin, respectively. The BET surface area and calculated total pore volume of LT were almost 10 times greater than that of CT suggesting that LT should provide more sites for sorption.
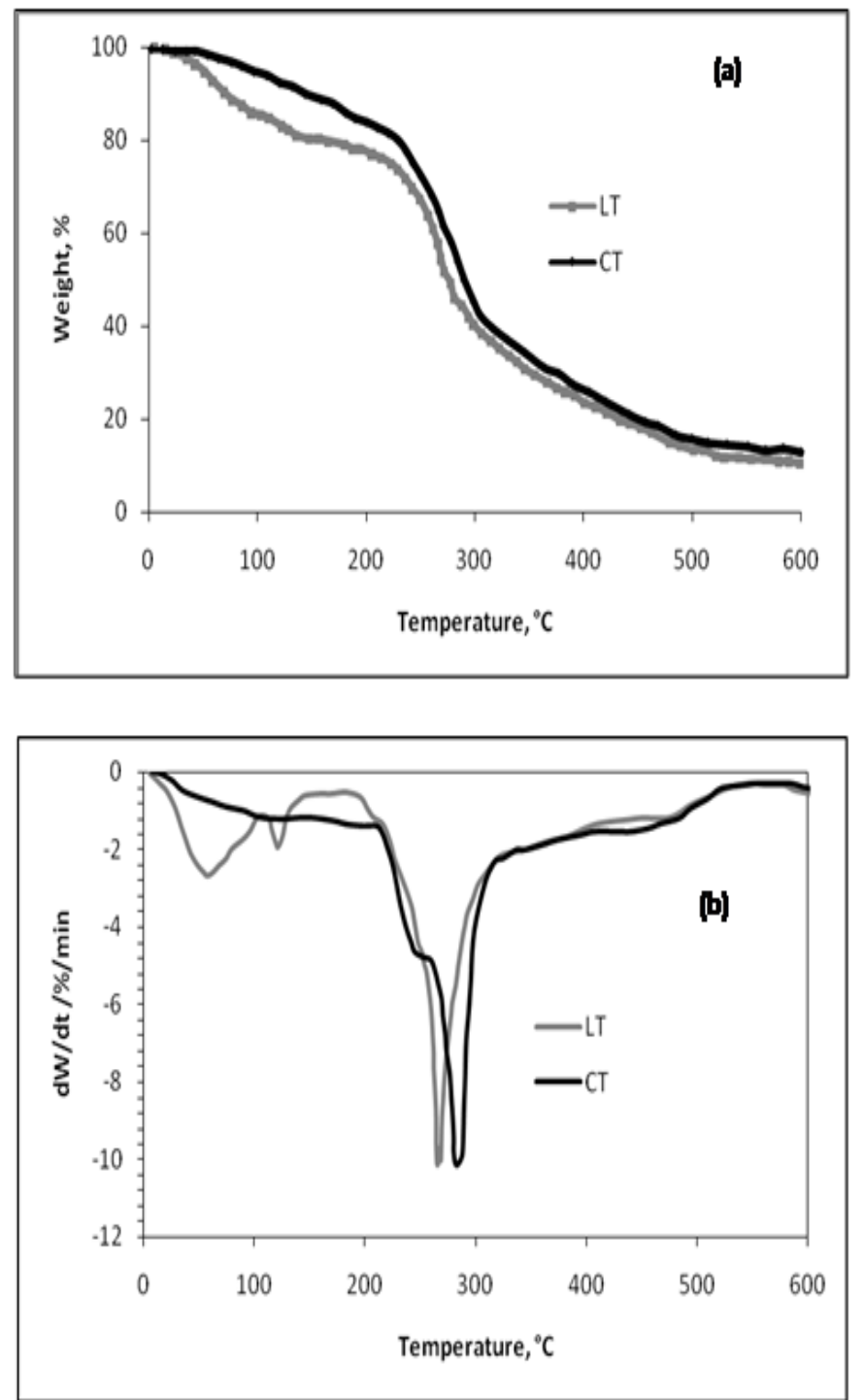

Figure 6 TGA (a) and DTG (b) curves of CT and LT.

\section{Synthesis and characterization of LRGR and LRFR}

Two modified resins; Resin-Tannin-Lyofilise-Glyoxal (RTLG) and Resin-Tannin Condensed-Glyoxal (RTCG) were synthesized. The gelation was studied by means of rheological measurements performed in an MCR 301 rheometer. The evolution of the viscosity with a shear rate of $1 \mathrm{~s}^{-1}$ during the curing process of RTCG and RTLG is shown in Figure 7. It is observed that although the time taken by the resin to reach the gel point varies with type of tannin, lyophilized tannin is more reactive than condensed tannin it has lower gelation time. In the case of condensed tannins (path 2), because of the low solubility in water of the fractions and the low reactivity of these tannins toward glyoxal, it was not possible to establish gel times. This behaviour could be both explained by the well know catechinic acid rearrangement (Figure 8$)^{20}$ and rearrangement of flavonoid to phlobatannins which would occur during the acidification step. ${ }^{21}$

Compared to acidified tannins, the higher reactivity of the lyophilized extract is probably due to the low catechinic acid content. ${ }^{22}$

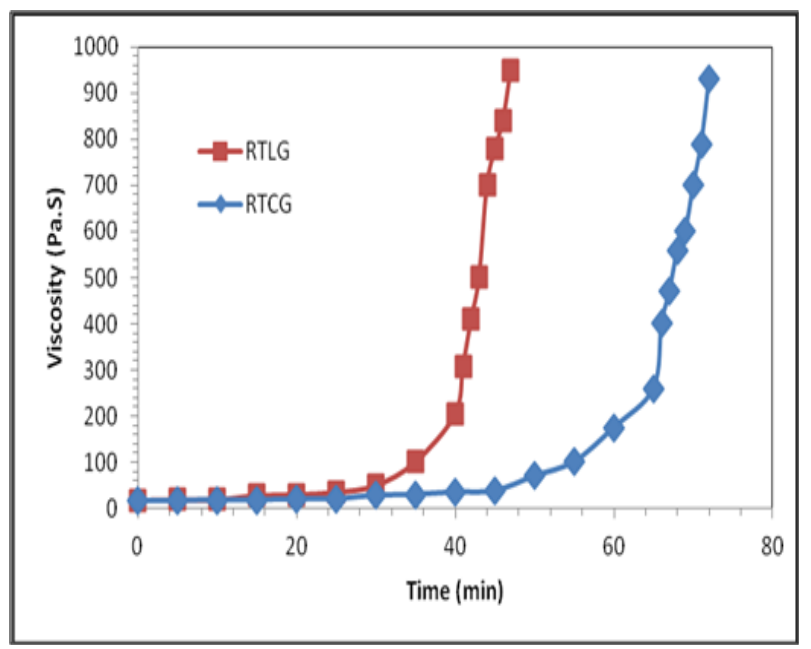

Figure 7 Evolution of viscosity during the curing process of RTLG and RTCG at cure temperature of $25^{\circ} \mathrm{C}$.

\section{Effect of temperature on gelation time}

The effect of temperature on the gelation time for polymer gel was studied since the $1980 \mathrm{~s}$, and yet, the temperature problem still attracts researchers..$^{23}$ The temperature is in equilibrium with its activated state for a gelation reaction in Equation (1). It is known that the gelant solution will degrade at the above critical temperature.

$$
T_{g e l}=\frac{\Delta E}{x \Delta S^{0}}
$$

Where $T_{g e l}$ is critical gelation temperature, $\Delta E$ corresponds to the difference between the energies of activation for reactions carried out at two different initial solution concentrations, $\Delta S^{\circ}$ is the standard state entropy difference between the products and reactants for a chemical reaction, and $\mathrm{x}$ is the power of the initial concentration.

The gelation reaction is strongly dependant on temperature. Figure 9 shows the effect of temperature on gelation time. As the temperature increases, the gelation time decreases quickly. With Condensed tannin (RTCL) the gelant solution begins to form in about 40 minutes if the solution is kept at room temperature. It is about $10-12 \mathrm{~min}$ if measured at the temperature of $80^{\circ} \mathrm{C}$.

Generally, the cross-linking reaction occurs due to the presence of the Hydroxyl groups in the polymer structure. Hence, the degree of hydroxide groups (IOH) can control the reaction.

The degree of hydroxyl groups is higher in RTLG than RTCG (Table 1) which explain the higher reactivity of lyophilized tannin compared to condensed tannin. When the temperature increases, more aromatic ortho and para carbon groups become available. In other words, the higher temperature reduces gelation time.

\section{Effect of $\mathrm{pH}$ on gelation time}

The $\mathrm{pH}$ of polymer solution influences the gelation time. There are only specific ranges of $\mathrm{pH}$ that perform well in the gelation rate. The 
$\mathrm{pH}$ value also affects the degree of hydroxyl group. In this research, the gel solution was mixed and then acid $\mathrm{HCl}$ or alkali $\mathrm{NaOH}$ was added to modify the initial $\mathrm{pH}$ value before the gelation time was measured by using a viscometer.

Figure 10 shows that the gelation time is reduced when $\mathrm{pH}$ changes from 6 to 11 and the viscosity of polymer solution increases<smiles>C=CC1C=CC(=CC(O)Cc2c(O)cc(O)cc2O)C=C1CC(O)C=CC</smiles>

\section{Catechin}

Figure 8 Mechanism of formation of catechinic acid.

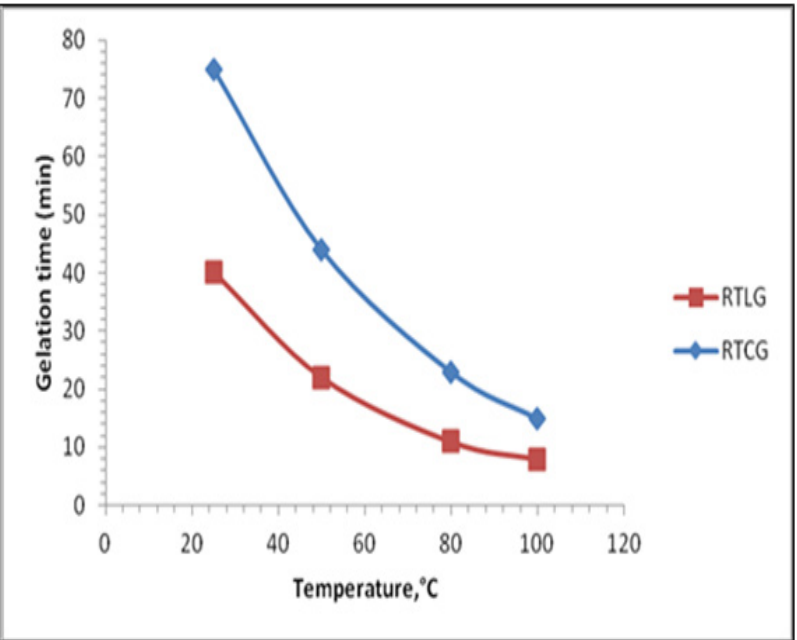

Figure 9 Gelation time of RTLG and RTCG was measured at different temperatures.

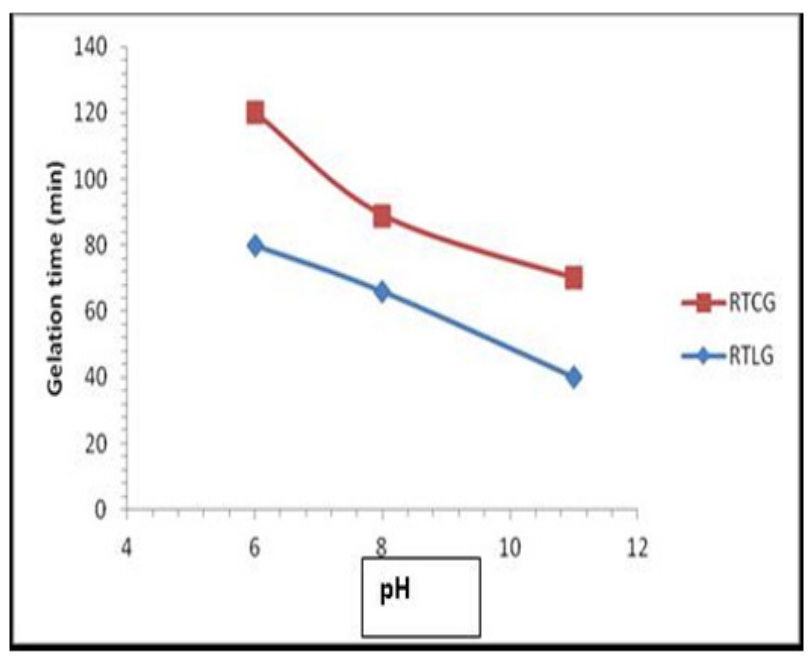

Figure 10 The gelation time versus initial $\mathrm{pH}$ for RTCG and RTLG. with increasing $\mathrm{pH}$. Above 6 the lower $\mathrm{pH}$ solutions are the longest gelation time. ${ }^{24}$

The gelation time decreases slightly until obtaining the value 11 . The best range of $\mathrm{pH}$ for the making of a gel solution is between 8 and 11 .

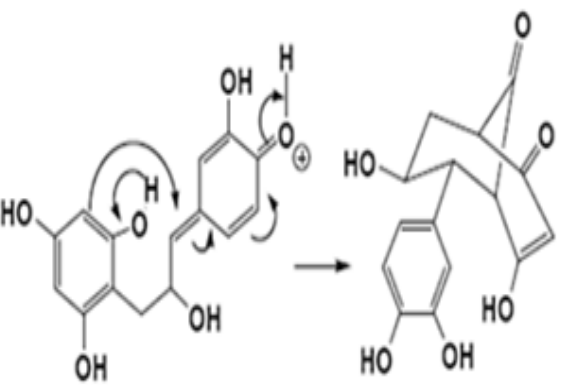

Catechinic acid

Table I Synthesis conditions in the preparation of Resin-Tannin-LyophilizeGlyoxal (RT-LG) and Resin-Tannin-Condensed-Glyoxal (RT-CG)

\begin{tabular}{llll}
\hline & $\mathbf{p H}$ & IOH mmol/g & $\%$ Glyoxal \\
\hline RTLG & II.2 & I.4 & 50 \\
RTCG & 9.1 & 0.8 & 50
\end{tabular}

Table 2 BET measurements of CT and LT

\begin{tabular}{llll}
\hline BET surface area & $\begin{array}{l}\text { Pore diameter } \\
(\mathbf{n m})\end{array}$ & $\begin{array}{l}\text { Total pore } \\
\text { volume }(10-4 \\
\left.\mathbf{c m}^{3} / \mathbf{g}\right)\end{array}$ \\
\hline CT & 3.2 & 3.8 & 0.7 \\
LT & 30.1 & 10.4 & 8.1 \\
\hline
\end{tabular}

\section{Effect of curing agent type on gelation time}

To study the effect of curing agent type, different solutions were mixed with the same concentration and ratio, only changing the curing agent type from glyoxal (RTLG) to formaldehyde (RTLF).

Figure 11 shows that the gelation of solutions, mixed with Glyoxal, has a longer gelation time than solutions mixed with formaldehyde. This means that formaldehyde is more reactive than glyoxal. It enhances the generation of prepolymers and helps the cross-linking reaction to occur faster. In the two cases when the temperature increase the gelation time decrease. ${ }^{25,26}$

\section{Thermal stability}

For comparing the thermal properties of the RTLG and RTCG resin fractions, thermo gravimetric analysis (TGA) was applied to determine the thermal stability and degradation of the two resins. The TGA curve presents the weight loss percentage of the sample. As illustrated in the Figure 12, the TGA curves of the two resin fractions exhibited three stages during the pyrolysis process. At the first stage, the temperature below $200^{\circ} \mathrm{C}$, the weight loss was due to the volatilization of moisture present in the resin samples as well as some decomposition products with low molecular weight, such as carbon 
dioxide, carbon monoxide, and methane. At the second stage, which involved a wide range of temperature from 200 to $500^{\circ} \mathrm{C}$, the main weight loss could be explained by the violent degradation of cured resin. At the third stage, when the temperature was beyond $500^{\circ} \mathrm{C}$, the weight loss was not very evident. At this stage, decomposition reactions often occurred in concurrence with condensation reactions of aromatic rings. ${ }^{27}$

The results demonstrated that the two resin fractions has the same thermal stability which indicate that the extraction process do not affect the thermal stability. According to Ammar et al. ${ }^{29}$ the thermal degradation of Lignin-Glyoxal resin occurs at $800^{\circ} \mathrm{C}$, which indicate that tannin resin have a lower thermal stability compared to lignin resin..$^{28,29}$

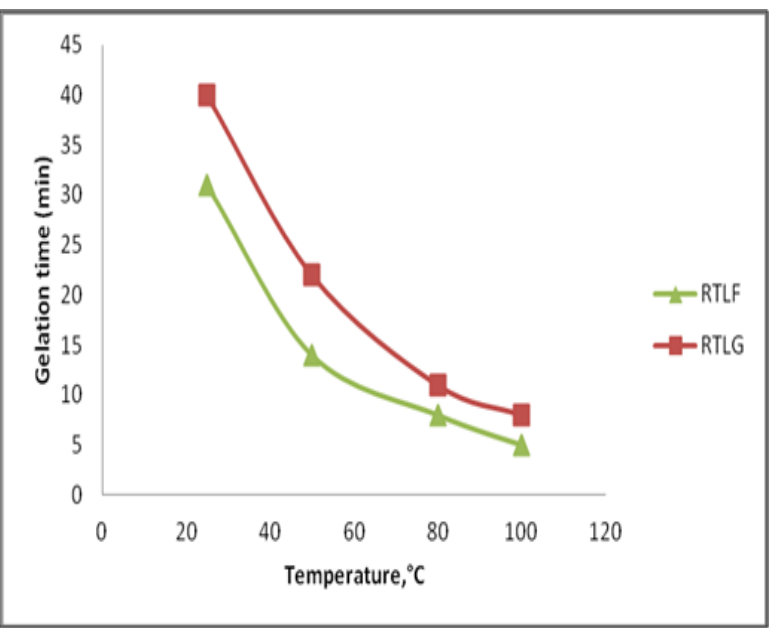

Figure I I Effect of curing agent in the gelation time.

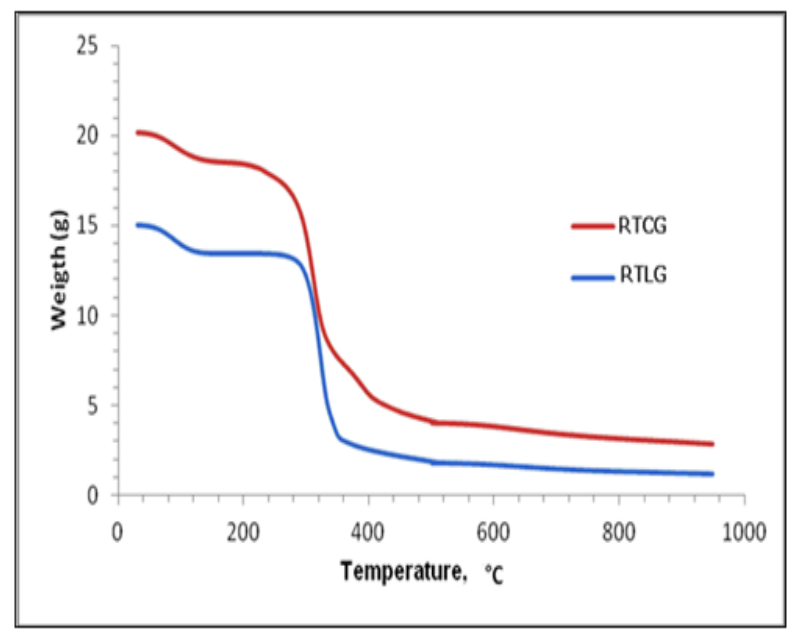

Figure 12 TGA Curves of RTCG and RTLG resin.

\section{Conclusion}

The conclusions from the results of the experiments in this study are as follows:

A. The gelation time can be controlled by changing the $\mathrm{pH}$ solution and temperature.

B. Lyophilized tannin is more reactive than condensed tannin it has lower gelation time. The two tannins fractions have the same thermal stability.
C. Adhesive reactivity of Tannin-formaldehyde was found to be better than Tannin-glyoxal resin: this reflects the copolymerization of tannin with formaldehyde resulting in better binding strength.

D. Furthermore, it can be said that the crosslinked tannin-glyoxal resin systems presented show potential for the production of polymers with high bio-base contents.

\section{Acknowledgements}

The authors express their grateful thanks and sincere gratitude to Prof. Mohamed Naceur Belgacem and the "PHC-UTIQUE CMCU" for their financial support.

\section{Conflict of interest}

No conflict of interest exists.

\section{References}

1. Harborne JB. Secondary plants products. Antinutritional and beneficial actions in animal feeding. Grass \& Forage Sci. 1999;55(4):378.

2. Schofield P, Mbugua DM, PellAN. Analysis of condensed tannins: a review. Anim Feed Sci Tech. 2001;91(1-2):21-40.

3. Van Soest PJ. Nutritional ecology of the ruminant. 2nd ed. Ithaca: Cornell Univ Press; 1994. p. 476.

4. Pizzi A. Wood adhesives: chemistry and technology. New York: Marcell Dekker; 1983. p. 364.

5. Clifford MN, Scalbert A. Ellagitannins-nature, occurrence and dietary burden. JJ Sci Food Agric. 2000;80(7):1118-1125.

6. He Q, Yao K, Sun D, et al. Biodegradability of tannin-containing wastewater from leather industry. Biodegradation. 2007;18(4):465-472.

7. Mateus N, Carvalho E, Luis C, et al. Influence of the tannin structure on the disruption effect of carbohydrates on protein tannin aggregates. Anal Chim Acta. 2004;513(1):135-140.

8. Pizzi A. Handbook of Adhesive Technolog. 2nd ed. Marcel Dekker Inc, New York; 2003. p. 999.

9. Pizzi A. Recent developments in eco-efficient bio-based adhesives for wood bonding: opportunities and issues. J Adhes Sci Technol. 2006;20(8):829-846.

10. Tondi G, Pizzi A. Tannin-based rigid foams: Characterization and modification. Ind Crop Prod. 2006;29(2-3):356-363.

11. Lei H, Pizzi A, Du G. Environmentally friendly mixed tannin/lignin wood resins. J Appl Polym Sci. 2008;107(1):203-209.

12. Panamgama LA. Polyphenolic Extracts of Pinus radiata Bark and networking Mechanisms of Additive-Accelerated Polycondensates. J Appl Polym Sci. 2007;103(4):2487-2493.

13. Fradinho DM, Pascoal Neto C, Evtuguin D, et al. Chemical characterisation of bark and of alkaline bark extracts from maritime pine grown in Portugal. Ind Crop Prod. 2002;16(1):23-32.

14. Lan P, Brosse N, Chrusciel L, et al. Extraction of condensed tannins from grape pomace for use as wood adhesives. Ind Crop Prod. 2011;33(1):253-257.

15. Ping L, Brosse N, Pizzi A, et al. Condensed tannins extraction from grape pomace: characterization and utilization as wood adhesives for wood Particleboard. Ind Crops Prod. 2012;34(1):907-914.

16. El Mansouri NE, Pizzi A, Salvado J. Lignin-based polycondensation resins for wood adhesives. J Appli Polym Sci. 2007;103(3):1690-1699. 
17. Ammar M, Khiari R, Belgacem MN, et al. Thermal characterization and comparisons of Lignin-Formaldehyde and Lignin-Glyoxal adhesives. Mediterranean J chem. 2014;2(6):731-737.

18. Hong Y, Subodh G, Mhaisalkar, et al. Observations of Gelation and Vitrification of a Thermosetting Resin during the Evolution of Polymerization Shrinkage. Macromol Rapid Commun. 2005;26(18):1483-1487.

19. Mortimer S, Ryan AJ, Stanford JL. Rheological Behavior and Gel-Poin Determination for a Model Lewis Acid-Initiated Chain Growth Epoxy Resin. Macromolecules. 2001;34(9):2973-2980.

20. Dean K, Cook WD, Rey L, et al. Near Infrared and Rheological Investigations of Epoxy-Vinyl Ester Interpenetrating Polymer Networks. Macromolecules. 2001;34(19):6623-6630.

21. Kiatgrajai P, Wellons JD, Gollob L, et al. Kinetics of Epimerization of (+)-Catechin and Its Rearrangement to Catechinic Acid. J Org Chem. 1982;47(15):2910-2912.

22. Sealy-Fisher VJ, Pizzi A. Increased pine tannins extraction and wood adhesives development by phlobaphenes minimization. Holz als Roh-und Werkstoff. 1992;50(5):212-220.

23. Amaral-LabatGA, Pizzi A, Goncalves AR, et al. Environment-Friendly Soy Flour-Based Resins without Formaldehyde. J Appl Polym Sci. 2008;108(1):624-632.
24. Jordan DS, Green DW, Terry RE, et al. The effect of temperature on gelation time for polyacrylamide/chromium (III) systems. SPE 56th Annual Technical Conference and Exhibition. San Antonio, Texas; 1982.

25. Choi SK, Sharma MM, Bryant SL, et al. $p H$-sensitive polymers for novel conformance control and polymerflood applications. SPE International Symposium on Oilfield Chemistry, Woodlands, Texas; 2009.

26. Mohammad S, Mohsen VS, Ahmad DK, et al. Polyacrylamide gel polymer as water shut-off system: Preparation and investigation of physical and chemical properties in one of the Iranian oil reservoirs conditions. Iran J Chem Chem Eng. 2007;26(4):99-108.

27. Nguyen NTB, Tu TN, Bae W, et al. Gelation Time Optimization for an HPAM/Chromium Acetate System: The Successful Key of Conformance Control Technology. Energy sources. Part A, Recovery, util. environ. Effects. 2012;34(14):1305-1317.

28. Hoong YB, Paridaha MT, Luqman CA, et al. Fortification of sulfited tannin from the bark of Acacia mangium withphenol-formaldehyde for use as plywood adhesive. Ind Crops Prod. 2009;30(3):416-421.

29. Ammar M, Khiari R, Belgacem $\mathrm{MN}$, et al. Isolation and characterization of lignin's from Stipa tenacissima L. and Phoenix dactylifera. Cellulose chemistry and technology. Cellulose Chem Technol. 2014;48(3-4):255-263. 